\title{
NC-6004 Phase I study in combination with gemcitabine for advanced solid tumors and population PK/PD analysis
}

\author{
Toshihiko Doi ${ }^{1}$ Tetsuya Hamaguchi ${ }^{2} \cdot$ Kohei Shitara $^{1} \cdot$ Satoru Iwasa $^{2} \cdot$ \\ Yasuhiro Shimada ${ }^{2} \cdot$ Mitsunori Harada $^{3} \cdot$ Kenichiro Naito $^{3} \cdot$ Naoto Hayashi $^{3}$. \\ Atsuhiro Masada ${ }^{3}$ (D) Atsushi Ohtsu ${ }^{1}$
}

Received: 12 December 2016 / Accepted: 6 February 2017 / Published online: 21 February 2017

(C) The Author(s) 2017. This article is published with open access at Springerlink.com

\begin{abstract}
Objectives This study was an open-label phase I study to confirm the safety and tolerability of NC-6004 in combination with gemcitabine in Japanese patients with advanced solid tumors and to assess the PK effects of NC-6004 monotherapy.

Methods This phase I study used a $3+3$ design to determine the maximum tolerated dose (MTD) and recommended dose of NC-6004 combined with gemcitabine. Safety and pharmacokinetics were assessed. The administration of NC-6004 alone was started at $60 \mathrm{mg} / \mathrm{m}^{2}$ every treatment cycle (21 days per cycle). From the second through eighth cycles, patients received NC-6004 in combination with $1000 \mathrm{mg} / \mathrm{m}^{2}$ of gemcitabine that was administered on day 1 and day 8 of each cycle, except for the first treatment cycle.

Results Twelve patients with advanced solid tumors received 60 or $90 \mathrm{mg} / \mathrm{m}^{2} \mathrm{NC}-6004$. Both MTD and RD were determined to be $90 \mathrm{mg} / \mathrm{m}^{2}$. The most common drugrelated adverse events were neutrophil decrease (66.7\%) and white blood cell count decrease (41.7\%). Population pharmacokinetic (PK) analysis revealed that NC-6004 PK profile in Japanese study was not significantly different from that in a previous Caucasian study.
\end{abstract}

Atsuhiro Masada

masada@nanocarrier.co.jp

1 Exploratory Oncology Research and Clinical Trial Center, National Cancer Center Hospital East, Chiba, Japan

2 Gastrointestinal Oncology Division, National Cancer Center Hospital, Tokyo, Japan

3 NanoCarrier Co. Ltd., Onoya Kyobashi Building, 1-4-10 Kyobashi, Chuo-ku, Tokyo 104-0031, Japan
Conclusions Both MTD and RD of NC-6004 were determined to be $90 \mathrm{mg} / \mathrm{m}^{2}$. The pharmacodynamic (PD) model well explained the time course of estimated glomerular filtration rate (eGFR) and amplitude of decrease in eGFR. The decrease in eGFR appeared to reach saturation at $>100 \mathrm{mg} / \mathrm{m}^{2}$ with NC-6004. Estimated probability of acute kidney injury on this PK/PD simulation was 30\% with NC-6004 and 70\% with cisplatin, which may better explain the renal toxicity profile.

Keywords Acute kidney injury · Cisplatin - Micelles · Population PK/PD analysis · NC-6004

$\begin{array}{ll}\text { Abbreviations } \\ \text { PK } & \text { Pharmacokinetic } \\ \text { AKI } & \text { Acute kidney injury } \\ \text { PD } & \text { Pharmacodynamic } \\ \text { eGFR } & \text { Estimated glomerular filtration rate } \\ \text { KDIGO } & \text { Kidney disease: improving global outcomes } \\ \text { DLT } & \text { Dose-limiting toxicity } \\ \text { Pt } & \text { Plasma total platinum } \\ \text { ICP } & \text { Inductively coupled plasma }\end{array}$

\section{Introduction}

NC-6004 is a novel micellar nanoparticle product of cisplatin, approximately $30 \mathrm{~nm}$ in diameter, obtained by crosslinking with a polyethylene glycol-poly glutamic acid block copolymer. Early basic research has indicated that when compared with conventional cisplatin, encapsulating cisplatin in the micellar nanoparticle can selectively improve accumulation at the tumor site. Indeed, NC-6004 progressively breaks down in the presence of chloride to release cisplatin slowly, and this slow release helps achieve 
longer systemic exposure that contributes to continuous and potentially improved antitumor effects. Cytotoxicity from NC-6004 is expected based on the formation of cellular DNA adducts with the released cisplatin.

Preliminary nonclinical studies have indicated several beneficial characteristics of NC-6004: (1) preferential distribution to tumors, (2) significantly lower toxicity compared with cisplatin at equivalent doses, and (3) increased antitumor activity [1]. The enhanced permeability and retention associated with polymeric micelles mean that the formulation benefited from extended blood circulation and selective and higher accumulation at the tumor site [2]. These clinical characteristics of NC-6004 have been demonstrated in both a phase I study where NC-6004 was used in monotherapy for patients with solid tumors in the UK (the NC-6004-001 study) and a phase I/II study where NC-6004 was used in combination with gemcitabine in patients with pancreatic cancer in Taiwan and Singapore (the NC-6004-002 study).

The present study was designed with two aims: (1) to assess the tolerability of NC-6004 in combination with gemcitabine in Japanese patients with solid tumors and (2) to obtain pharmacokinetic (PK) and pharmacodynamic (PD) data for NC-6004 monotherapy in Japanese patients.

\section{Materials and methods}

\section{Study design}

This study was an open-label phase I study to confirm the safety and tolerability of NC-6004 in combination with gemcitabine in Japanese patients with advanced solid tumors and to assess the PK effects of NC-6004 monotherapy. The study schedule comprised a screening phase, an NC-6004-alone phase, an NC-6004-plus-gemcitabine combination phase, and a follow-up phase. The study was conducted according to the provisions of the Declaration of Helsinki and the Guidelines for Good Clinical Practice, and was approved by the relevant institutional review board. Written informed consent was obtained from all patients before inclusion.

\section{Patient selection}

Japanese patients with treatment-refractory solid tumors were recruited. Inclusion criteria were as follows: (1) age between 20 and 75 years, (2) histologically or cytologically confirmed cancer, (3) evaluable tumor lesions according to the Response Evaluation Criteria in Solid Tumors guideline (Version 1.1), (4) Eastern Cooperative Oncology Group performance status 0 to 2 , and (5) adequate bone marrow reserve at screening. The exclusion criteria were as follows: (1) known hypersensitivity to platinum compounds or gemcitabine; (2) previous therapy with more than two different platinumbased regimens, or a regimen with a cumulative dose exceeding $480 \mathrm{mg} / \mathrm{m}^{2}$ for cisplatin, $1040 \mathrm{mg} / \mathrm{m}^{2}$ for oxaliplatin, or $42 \mathrm{mg} / \mathrm{mL} / \mathrm{min}$ for carboplatin (cumulative area under the curve); (3) previous chemotherapy or radiotherapy within 28 days before the study treatment; (4) a history of symptomatic pulmonary fibrosis or interstitial pneumonia, with obvious evidence on a plain X-ray of the chest; (5) previous chest radiotherapy; (6) diagnosed chronic kidney disease defined as an estimated glomerular filtration rate (eGFR) $<60 \mathrm{~mL} /$ $\mathrm{min} / 1.73 \mathrm{~m}^{2}$; and (7) greater than grade 2 auditory toxicity by pure tone audiometry or greater than grade 2 neurotoxicity.

\section{Dosing rationale}

Although the MTD was not determined during the NC-6004-001 phase I study in the UK, the RD was suggested to be close to $90 \mathrm{mg} / \mathrm{m}^{2}$ for monotherapy. The RD of NC-6004 given with gemcitabine has also been estimated to be $90 \mathrm{mg} / \mathrm{m}^{2}$ based on the phase I part of the NC-6004-002 study in Asia. From those results, the RD of NC-6004 in the Japanese patient population has been estimated at $90 \mathrm{mg} /$ $\mathrm{m}^{2}$; however, in this study we initiated dosing at $60 \mathrm{mg} / \mathrm{m}^{2}$, and NC-6004 was mixed in $250 \mathrm{~mL}$ of $5 \%$ dextrose solution and given intravenously for over 60 min daily for 21 days. Oral dexamethasone; normal saline solution, including $\mathrm{KCl}$ and $\mathrm{MgSO} 4$; diphenhydramine hydrochloride; ranitidine; and mannitol were also provided as a prophylactic treatment for hypersensitivity before and after each treatment cycle. Treatment could be continued until eight cycles over 24 weeks, with the patient observed until 28 days after the eighth cycle or until discontinuation of the study treatment.

\section{Protocol: determination of the MTD and RD}

The administration of NC-6004 alone was started at $60 \mathrm{mg} /$ $\mathrm{m}^{2}$ every treatment cycle (21 days per cycle). From the second through eighth cycles, patients received NC-6004 in combination with $1,000 \mathrm{mg} / \mathrm{m}^{2}$ of gemcitabine that was administered on day 1 and day 8 of each cycle, except for the first treatment cycle.

Three patients were expected to be enrolled in the NC-6004 $60 \mathrm{mg} / \mathrm{m}^{2}$ cohort. When a dose-limiting toxicity (DLT) event was observed, another three patients would be added to assess safety. A maximum of nine patients could be enrolled, as necessary, to obtain further safety information in this cohort. If two of the six or nine patients reached a DLT event at $60 \mathrm{mg} / \mathrm{m}^{2}$, that was considered the MTD. After evaluating safety for all patients, including at least two cycles of the study treatment, the first patient in the next $90 \mathrm{mg} / \mathrm{m}^{2}$ cohort was enrolled. Subsequent dose escalations were planned to doses of 120 or $150 \mathrm{mg} / \mathrm{m}^{2}$ in the 
same manner. The MTD was defined as the dose at which two of the six or nine patients experienced DLT. The RD was defined as the dose one level lower than the MTD; however, in all cases, the MTD or RD was only confirmed after discussion between the sponsor and the medical expert.

DLT was defined as follows: (1) grade 4 hematologic toxicity; (2) grade 3 thrombocytopenia with bleeding; (3) grade 3 or greater neutropenia with fever over $38.5^{\circ} \mathrm{C}$ or with grade 2 or greater diarrhea; (4) grade 3 or greater neutropenia without fever for at least five days; (5) grade 3 or greater non-hematologic toxicity (except alopecia, treatable nausea or emesis, and biochemistry abnormalities without specific symptoms); (6) treatment delay of greater than two weeks before the start of the next treatment cycle (NC-6004 or gemcitabine) due to unresolved toxicity; (7) grade 3 or greater hypersensitivity reaction; (8) an eGFR toxicity of $30-59 \mathrm{~mL} / \mathrm{min} / 1.73 \mathrm{~m}^{2}$ delaying dosing by more than 14 days; or (9) an eGFR $<30 \mathrm{~mL} / \mathrm{min} / 1.73 \mathrm{~m}^{2}$, or apparent renal failure identified by another index.

\section{Statistical analysis}

Data were not tested statistically because this phase I study focused on safety and tolerability, estimation of the RD, and collection of PK data, except in the case of the population analysis.

\section{Population analysis}

All population analyses were performed by NONMEM ver. 7.3.0, using the FOCE INTER estimation method. A difference of 7.879 in the value of the objective function $(\triangle \mathrm{OFV})$ with one degree of freedom was defined as being statistically significant $(P<0.005)$. A proportional model was selected for the residual error and inter-individual variability for the stochastic model.

\section{Population PK analysis}

Blood samples for PK analysis were taken immediately before dosing, just after dosing, and at 1, 3, 6, 24, 48, 72, 168,336 , and $504 \mathrm{~h}$ after dosing. A population PK analysis was then performed using data obtained in this study that were merged with those from the phase I study performed by NanoCarrier Co., Ltd. in the UK (NC-6004-001 study).

The plasma total platinum (Pt) concentration was measured by the inductively coupled plasma (ICP) method in this study. In the previous UK study, plasma total Pt concentration was measured by both ICP and atomic absorption spectroscopy (AA) methods. Outlier data were not excluded from the analysis. In this PK analysis, the ratio of values between ICP and AA had an intra-individual distribution and the AA value was converted to an ICP value.
Two compartments with a first-order elimination model were selected for PK structural model. A proportional model was selected for the residual error and inter-individual variability as the stochastic model.

As the possible/available covariates, baselines of body weight and eGFR and ethnic difference were selected. Clearance and volume of distribution might be influenced by these covariates.

First, all possible combinations of covariates and PK parameters were included in the base model, and non-significant covariates were then excluded. The models including all significant covariates were tested for each covariate, and the significant covariates remained in the final model.

\section{Population PK/PD analysis}

A population PK/PD model for eGFR was built with an empirical indirect model of Model I [3], using the following equation for the eGFR time course:

$\frac{d e G F R}{d t}=k_{\text {in }} \times\left(1-\frac{I_{\max } \times C_{f}}{I C_{50}+C_{f}}\right)-k_{\text {out }} \times e G F R$,

where $\mathrm{GFR}_{0}$ is the baseline GFR, $I_{\text {max }}$ is the maximum inhibition $\left(0<I_{\max }<1\right), \mathrm{k}_{\text {in }}$ is eGFR $\mathrm{Gr}_{0} \times K_{\text {out }}, \mathrm{IC}_{50}$ is the concentration to achieve $50 \%$ inhibition, and $C_{\mathrm{f}}$ is the plasmafree Pt concentration.

Individual $\mathrm{C}_{\mathrm{f}}$ was derived from population $\mathrm{PK}$ analysis. Most of the PK/PD parameters were assumed to be proportional to $\exp (\eta)$ with $\eta$ assumed to be normal with a mean of 0 . As shown in Fig. 1, the amplitude of the eGFR decrease after starting NC-6004 seemed to reduce with repeated administration. Therefore, $I_{\max }$ was assumed to decrease through repeated administrations and was expressed as follows:

$I_{\text {maxijk }}=I_{\max 0} \times \exp \left(-\operatorname{trend} \times t_{j}\right) \times \exp \left(\eta_{\mathrm{ik}}^{\operatorname{IOv}\left(I_{\text {max }}\right)}\right)$,

where $i, j$, and $\mathrm{k}$ represent the subject, measurement time, and occasion (i.e., the period through $k$ th to $[k+1]$ th administration). Inter-occasional variability (IOV) was also assumed because some patients showed the largest eGFR decrease after a later administration rather than after the first administration.

During and after model building, simulations were done. The serum creatinine $(\mathrm{sCr})$ simulation was done by calculating the eGFR using the Cockcroft-Gault equation in both clinical studies, with the increase of $\mathrm{sCr}$ ratio from baseline being required to be the same as the decrease in the eGFR ratio within a subject (i.e., an inverse relation within a patient). The mean and standard deviation for the baseline $\mathrm{sCr}$ in the NC-6004-003 study $(0.772 \pm 0.124 \mathrm{mg} / \mathrm{dL})$ were 

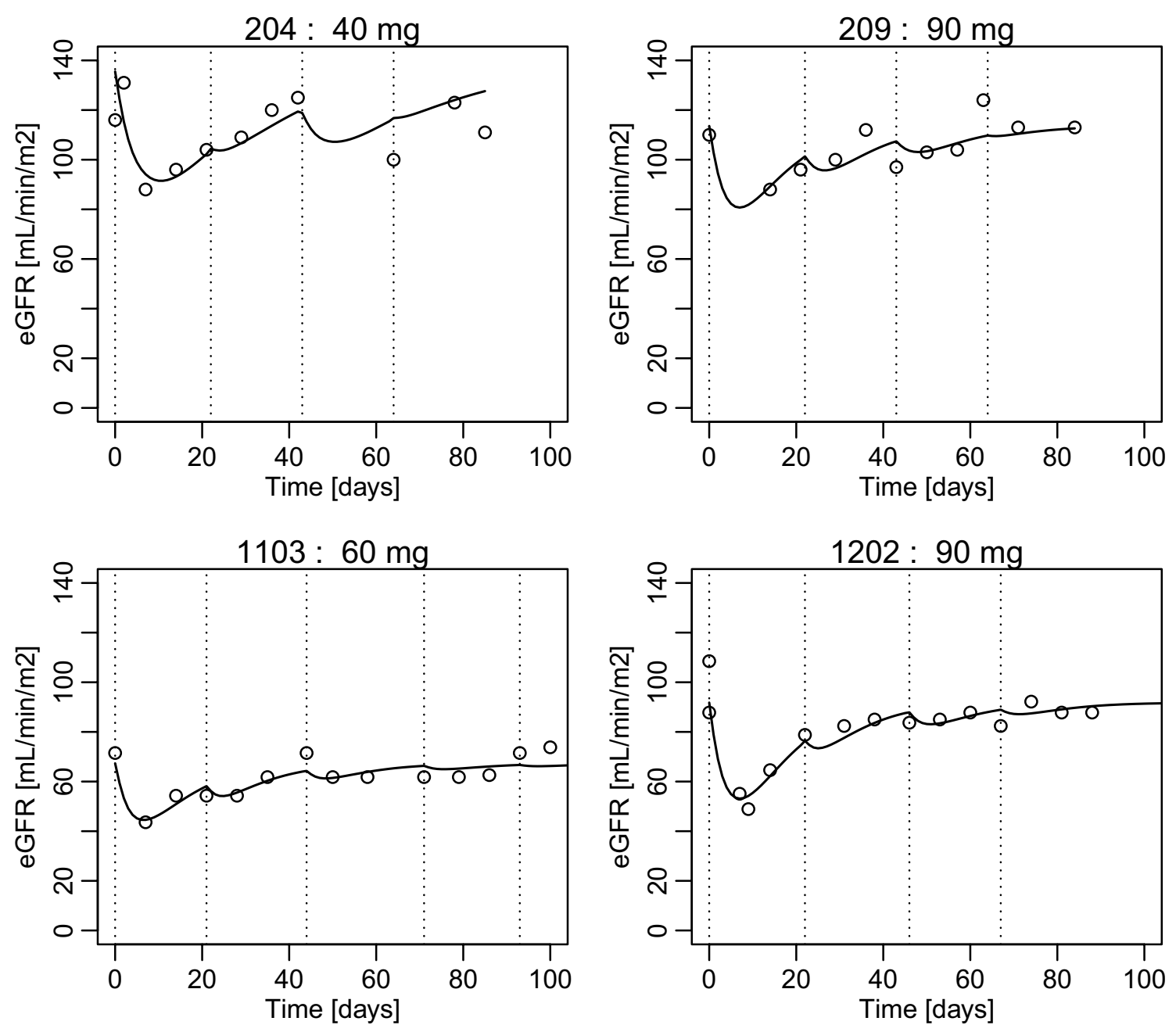

Fig. 1 Example of individual time course for the estimated glomerular filtration rate. Circle shows the observed value, and curve shows the model-predicted time course. Dotted line shows the administra-

used for the sCr simulation on day 7, which was 6 days after the first administration.

Renal dysfunction severity was calculated for each virtual subject according to the Kidney Disease: Improving Global Outcomes (KDIGO) definition: stage $1=0.3 \mathrm{mg} / \mathrm{dL}$ increase from or 1.5-1.9 times the pretreatment value; stage $2=2.0-2.9$ times the pretreatment value; and stage $3=\geq 3.0$ times the pretreatment value or the $\mathrm{sCr}$ increase $>4.0 \mathrm{mg} / \mathrm{dL}$ from the pretreatment value [4].

\section{Results}

\section{Patient characteristics}

The patients' demographics are summarized in Table 1 . The mean age of the ten male and two female patients was tion of NC-6004 (dotted lines administration). eGFR estimated glomerular filtration rate

59.5 years, ranging from 34 to 72 years. In total, seven patients had carcinoma and five patients had neuroendocrine tumor. Eight patients were classified as stage IV, and four patients were not classified. All patients had metastatic lesions. The most common site of metastasis was the lymph nodes, followed by the liver. All patients had a previous history of chemotherapy, and seven patients had received cisplatin treatment. One patient had undergone a previous gemcitabine treatment. The maximum cumulative dose of cisplatin was $420 \mathrm{mg} / \mathrm{m}^{2}$. Six patients had a history of surgery, and three had a history of radiotherapy.

\section{Safety analysis}

Patients were planned to receive NC-6004 at the doses of $60,90,120$, and $150 \mathrm{mg} / \mathrm{m}^{2}$ but were actually treated with only 60 and $90 \mathrm{mg} / \mathrm{m}^{2}$ doses because of the development 
Table 1 Patient demographics (safety population)

\begin{tabular}{|c|c|c|c|}
\hline \multirow[t]{2}{*}{ Characteristics } & \multicolumn{2}{|l|}{ Cohort } & \multirow[t]{2}{*}{ Total $(\%)$} \\
\hline & $60 \mathrm{mg} / \mathrm{m}^{2}(\%)$ & $90 \mathrm{mg} / \mathrm{m}^{2}(\%)$ & \\
\hline \multicolumn{4}{|l|}{ Age (year) } \\
\hline Number & 3 & 9 & 12 \\
\hline Mean \pm SD & $46.3 \pm 13.7$ & $63.9 \pm 7.5$ & $59.5 \pm 11.7$ \\
\hline Median & 44 & 66 & 65 \\
\hline Range & $34-61$ & $46-72$ & $34-72$ \\
\hline \multicolumn{4}{|l|}{ Gender } \\
\hline Male & $2(66.7)$ & $8(88.9)$ & $10(83.3)$ \\
\hline Female & $1(33.3)$ & $1(11.1)$ & $2(16.7)$ \\
\hline \multicolumn{4}{|l|}{ Tumor type } \\
\hline Sarcoma & $0(0.0)$ & $0(0.0)$ & $0(0.0)$ \\
\hline Carcinoma & $1(33.3)$ & $6(66.7)$ & $7(58.3)$ \\
\hline Lymphoma & $0(0.0)$ & $0(0.0)$ & $0(0.0)$ \\
\hline Other & $2(66.7)$ & $3(33.3)$ & $5(41.7)$ \\
\hline \multicolumn{4}{|c|}{ Metastatic sites (multi-choice) } \\
\hline Adrenal & $0(0.0)$ & $0(0.0)$ & $0(0.0)$ \\
\hline Liver & $3(100.0)$ & $4(44.4)$ & $7(58.3)$ \\
\hline Bone & $1(33.3)$ & $0(0.0)$ & $1(8.3)$ \\
\hline Lymph nodes & $3(100.0)$ & $5(55.6)$ & $8(66.7)$ \\
\hline Lung & $1(33.3)$ & $2(22.2)$ & $3(25.0)$ \\
\hline Skin/soft tissue & $0(0.0)$ & $0(0.0)$ & $0(0.0)$ \\
\hline Other & $1(33.3)$ & $4(44.4)$ & $5(41.7)$ \\
\hline N/A & $0(0.0)$ & $0(0.0)$ & $0(0.0)$ \\
\hline \multicolumn{4}{|l|}{ Stage } \\
\hline III & $0(0.0)$ & $0(0.0)$ & $0(0.0)$ \\
\hline IIIA & $0(0.0)$ & $0(0.0)$ & $0(0.0)$ \\
\hline IIIB & $0(0.0)$ & $0(0.0)$ & $0(0.0)$ \\
\hline IV & $3(100.0)$ & $5(55.6)$ & $8(66.7)$ \\
\hline \multicolumn{4}{|c|}{ Previous cancer treatment } \\
\hline \multicolumn{4}{|l|}{ Surgery } \\
\hline No & $2(66.7)$ & $4(44.4)$ & $6(50.0)$ \\
\hline Yes & $1(33.3)$ & $5(55.6)$ & $6(50.0)$ \\
\hline \multicolumn{4}{|l|}{ Chemotherapy } \\
\hline No & $0(0.0)$ & $0(0.0)$ & $0(0.0)$ \\
\hline Yes & $3(100.0)$ & $9(100.0)$ & $12(100.0)$ \\
\hline \multicolumn{4}{|l|}{ Radiotherapy } \\
\hline No & $2(66.7)$ & $7(77.8)$ & $9(75.0)$ \\
\hline Yes & $1(33.3)$ & $2(22.2)$ & $3(25.0)$ \\
\hline \multicolumn{4}{|l|}{ Other therapies } \\
\hline No & $3(100.0)$ & $8(88.9)$ & $11(91.7)$ \\
\hline Yes & $0(0.0)$ & $1(11.1)$ & $1(8.3)$ \\
\hline \multicolumn{4}{|c|}{ ECOG performance status } \\
\hline 0 & $2(66.7)$ & $5(55.6)$ & 7 (58.3) \\
\hline 1 & $1(33.3)$ & $4(44.4)$ & $5(41.7)$ \\
\hline 2 & $0(0.0)$ & $0(0.0)$ & $0(0.0)$ \\
\hline \multicolumn{4}{|l|}{ Hydration } \\
\hline With & - & $5(55.6)$ & $5(41.7)$ \\
\hline Without & $3(100.0)$ & $4(44.4)$ & $7(58.3)$ \\
\hline
\end{tabular}

ECOG Eastern Cooperative Oncology Group of the grade 3 or 4 drug-related toxicities, as shown in Table 2. Grade 3 or greater adverse events (AEs) related to NC-6004 included a neutrophil count decrease in the (two patients: $66.7 \%$ ) $60 \mathrm{mg} / \mathrm{m}^{2}$ cohort and (six patients: $66.7 \%$ ) $90 \mathrm{mg} / \mathrm{m}^{2}$ cohort and a decreased white blood cell count in the (four patients: $44.4 \%$ ) $90 \mathrm{mg} / \mathrm{m}^{2}$ cohort. At least one $\mathrm{AE}$ related to $\mathrm{NC}-6004$ was observed in all 12 patients in the safety evaluation cohort. Events in which the incidence was more than $40 \%$ were neutropenia (10 patients: $83.3 \%$ ), leukopenia (10 patients: $83.3 \%$ ), and thrombocytopenia (nine patients: $75.0 \%$ ).

The DLTs in each cohort were counted during the NC-6004 monotherapy phase (cycle 1) and the NC6004-plus-gemcitabine combination phase (cycle 2). No DLTs occurred in the $60 \mathrm{mg} / \mathrm{m}^{2}$ cohort of three patients, but four DLTs were observed in the $90 \mathrm{mg} / \mathrm{m}^{2}$ cohort of nine patients. In total, four of 12 patients experienced DLT: three patients experienced grade 4 hematologic toxicities, and one patient experienced a non-hematologic toxicity (an eGFR $<30 \mathrm{~mL} / \mathrm{min} / 1.73 \mathrm{~m}^{2}$ ). After observing the effect on eGFR, the study protocol was amended to add prophylactic hydration therapy [5] before and after treatment with NC-6004 to prevent renal toxicity. Seven patients did not receive hydration therapy, and other five patients did. No renal toxicity occurred among the patients who received hydration therapy. Based on these findings, the MTD and $\mathrm{RD}$ of NC-6004, when given in combination with gemcitabine, were both determined to be $90 \mathrm{mg} / \mathrm{m}^{2}$, with the latter being decided after discussion with the study sponsor.

\section{Efficacy analysis}

One patient showed partial response and eight patients had stable disease. The objective response rate and disease control rate were 9.1 and $81.8 \%$, respectively. Patient survival beyond 18 months after the first dose was analyzed by Kaplan-Meier survival curves. The curve reached a 50\% survival level by day 327, which persisted until day 596 (i.e., the final follow-up day).

\section{Population PK analysis}

The number of subjects included for PK analysis was 12 from this study and 17 from the UK study, resulting in a total of 546 observed concentrations for the final analysis. The total plasma Pt concentration was measured by the ICP method in all 12 patients in this study (108 points), while in the UK study total plasma Pt concentration was measured by the ICP method in five patients ( 42 points) and by the AA method in 17 patients (144 points). Notably, in the five patients whose total plasma Pt concentration was measured by both ICP and AA, the AA values were almost $70 \%$ higher than the ICP values, so the data obtained by 
Table 2 Grade 3 or 4

NC-6004-related adverse events

\begin{tabular}{llll}
\hline MedDRA SOC & MedDRA PT & $60 \mathrm{mg} / \mathrm{m}^{2}, N=3$ & $90 \mathrm{mg} / \mathrm{m}^{2}, N=9$ \\
\hline Investigations & Gamma-glutamyltransferase decreased & $0(0.0)$ & $1(11.1)$ \\
Investigations & Neutrophil count decreased & $2(66.7)$ & $6(66.7)$ \\
Investigations & Platelet count decreased & $1(33.3)$ & $1(11.1)$ \\
Investigations & White blood cell count decreased & $1(33.3)$ & $4(44.4)$ \\
$\begin{array}{l}\text { Metabolism and } \\
\text { nutrition disorders }\end{array}$ & Hypokalemia & $0(0.0)$ & $1(11.1)$ \\
\hline
\end{tabular}

MedDRA Medical Dictionary for Regulatory Activities, SOC System Organ Class, $P T$ preferred terms the AA method were converted to ICP values. The plasmafree $\mathrm{Pt}$ concentration was also measured in 28 patients $(252$ points). The selected population PK model was confirmed to explain the NC-6004 PK profile (free concentration also showed a good fit, but the data are not shown).

The PK parameters in the final model are summarized in Table 3. During covariate selection, the influence of ethnic difference on clearance was evaluated to have a $\triangle \mathrm{OFV}$ of $0.014(P=0.906)$. However, the influences of body weight on the clearance and central volume of distribution were significant. Clearance and central volume of distribution increased in proportion to around the 0.7 th power for body weight increase, as observed with other drugs. This has also become a good rationale of dose adjustment by body surface area. The typical central volume of distribution was $2.69 \mathrm{~L}$, which was smaller than the blood volume. Therefore, most of the administered NC-6004 was thought to be captured within vessels just after administration. The peripheral volume of distribution was $3.91 \mathrm{~L}$ and the amount of NC-6004 transferred to peripheral tissue was considered to be limited. Therefore, this drug should be barely transferable to some vulnerable tissues. Finally, the clearance of NC-6004 was estimated at $0.0687 \mathrm{~L} \mathrm{~h}$, corresponding to $1 / 100$ th of the normal GFR value, meaning that $\mathrm{NC}-6004$ should be stable in the human body.

\section{Population PK/PD analysis}

The estimated population PK/PD parameters are shown in Table 4. The number of subjects for the PK/PD analysis was 12 (mean 8.1 points/subject) from this study and 17
Table 3 Estimated population PK parameters (the final model)

\begin{tabular}{|c|c|c|}
\hline Parameter & Population mean & $\begin{array}{l}\text { Inter-individual } \\
\text { variability } \\
(\mathrm{CV} \%)\end{array}$ \\
\hline \multicolumn{3}{|l|}{ Inter-individual variability } \\
\hline $\mathrm{TV}^{\mathrm{a}}(\mathrm{L})$ & $2.69(2.39-2.99)$ & $13.9(7.3-18.2)$ \\
\hline$\Theta_{(\mathrm{V} 1-\mathrm{WT})}: \mathrm{V} 1=\mathrm{TV} 1 \times(\mathrm{WT} / 67.8)^{\Theta}$ & $0.763(0.412-1.11)$ & - \\
\hline $\mathrm{V}^{\dagger}(\mathrm{L})$ & $3.91(2.80-5.02)$ & - \\
\hline CL (L/h) & $0.0687(0.0615-0.0759)$ & $17.2(8.9-22.7)$ \\
\hline$\Theta_{(\mathrm{CL}-\mathrm{WT})}: \mathrm{CL}=\mathrm{TCL} \times(\mathrm{WT} / 67.8)^{\Theta}$ & $0.731(0.346-1.115)$ & - \\
\hline$Q(\mathrm{~L} / \mathrm{h})$ & $0.0117(0.0096-0.0138)$ & $20.1(0-28.5)$ \\
\hline Vm1 (L) & $81.6(69.7-93.5)$ & $24.4(0-36.5)$ \\
\hline $\mathrm{Vm} 2(\mathrm{~L})$ & $665(549-781)$ & $42.4(24.2-54.9)$ \\
\hline $\operatorname{CLm}(\mathrm{L} / \mathrm{h})$ & $6.54(5.56-7.52)$ & $45.7(21.8-60.9)$ \\
\hline Qm (L/h) & $9.30(5.73-12.87)$ & $54.4(22.2-73.7)$ \\
\hline Difference by method [folds] (AA to ICP) & $1.69(1.41-1.97)$ & $21.2(4.8-29.6)$ \\
\hline \multicolumn{3}{|l|}{ Intra-individual variability } \\
\hline Total Pt (ICP) (CV\%) & $17.0(15.2-18.7)$ & \\
\hline Total Pt (AA) (CV\%) & $31.6(27.5-35.3)$ & \\
\hline Free Pt (ICP) (CV\%] & $31.6(24.0-37.7)$ & \\
\hline
\end{tabular}

Mean (90\% CI)

$C L$ clearance, $V 1$ central volume of distribution, $V 2$ volume of distribution, $C V \%$ coefficient of variation, $I C P$ inductively coupled plasma method, $A A$ atomic absorption spectroscopy

${ }^{\text {a }}$ The typical value at $67.8 \mathrm{~kg}$ of body weight 
Table 4 Estimated population pharmacokinetic/pharmacodynamic model parameters

\begin{tabular}{lcl}
\hline Parameter & Population mean & $\begin{array}{l}\text { Inter-individual } \\
\text { variance }(\mathrm{CV} \%)\end{array}$ \\
\hline $\mathrm{GFR}_{0}\left(\mathrm{~mL} / \mathrm{min} / 1.73 \mathrm{~m}^{2}\right)$ & 83.4 & 26.5 \\
$K_{\text {out }}(/ \mathrm{day})$ & 0.171 & 48.7 \\
$\mathrm{IC}_{50}(\mathrm{ng} / \mathrm{mL})$ & 35.3 & 211.7 \\
$I_{\max 0}$ & 0.851 & - \\
$I_{\max }$ decrease trend $(/$ day $)$ & 0.0273 & - \\
$\mathrm{IOV}\left(I_{\max }\right)(\mathrm{CV} \%)$ & & 53.1 \\
Intra-individual variance $(\mathrm{CV} \%)$ & & 8.8 \\
\hline
\end{tabular}

$G F R_{0}$ baseline GFR; $I_{\max }$ maximum inhibition $\left(0<I_{\max }<1\right)$; $I C_{50}$ the concentration to achieve 50\% inhibition; GFR, glomerular filtration rate (mean 13.7 points/subject) from the UK study (NC-6004001 study).

The decrease in eGFR (\%) distribution per dose level was simulated and compared with the observed eGFR decrease. As shown in Fig. 2, most observed values were captured within $80 \%$ confidence intervals (CIs). The estimated $K_{\text {out }}$ suggests that the injured kidney function (eGFR) recovered to half the damage level by 4 days and that there was a relatively large difference in this recovery speed among patients [inter-individual coefficient of variation $(\mathrm{CV} \%)$ of $48 \%$ ].

The population mean of $\mathrm{IC}_{50}$ (i.e., the concentration of free $\mathrm{Pt}$ that causes half of the maximum damage) was estimated at $35.3 \mathrm{ng} / \mathrm{mL}$. There was a huge variability in the
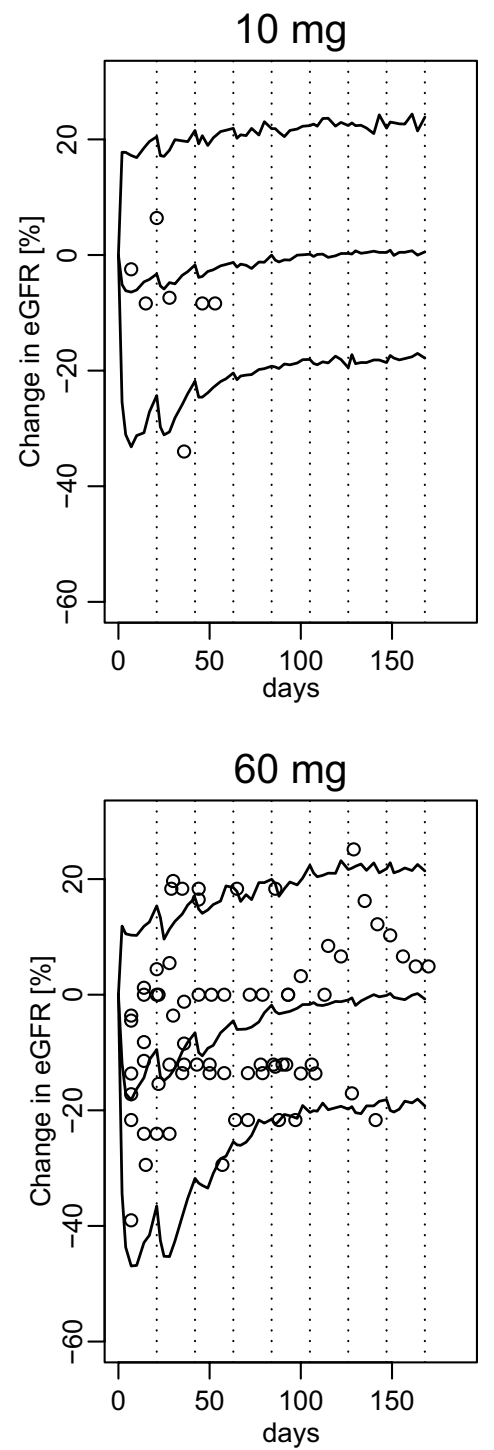
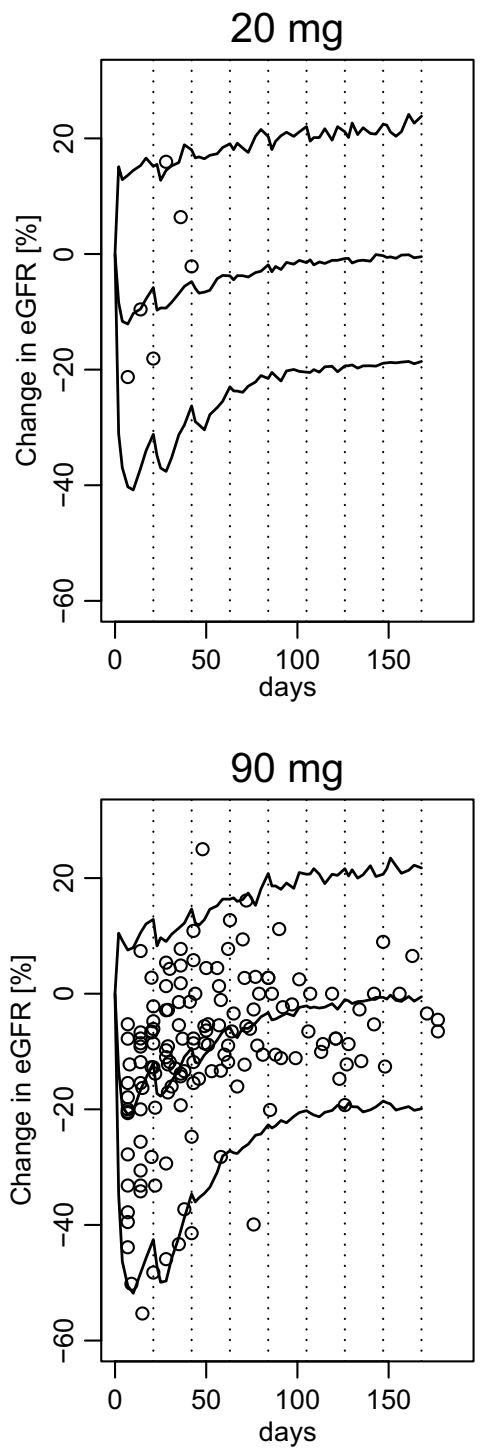
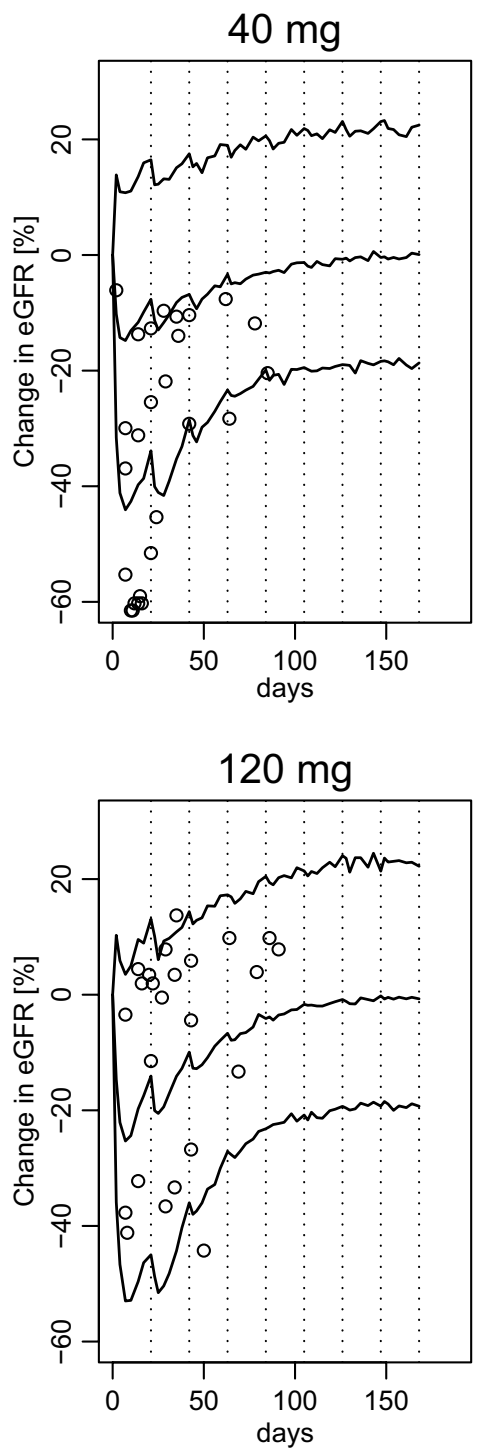

Fig. 2 Model prediction showing 80\% confidence intervals (CIs) for change in estimated glomerular filtration rate and the observed values. Curves show $90 \%$ and $10 \%$ quintiles and the median simulated values, with the dotted lines showing the administration timings. Abbreviations: eGFR, estimated glomerular filtration rate 
$\mathrm{IC}_{50}$ value, with a CV\% of 211.7 , meaning that there was a very broad range of susceptibility to free Pt exposure. The $\mathrm{IC}_{50}$ and $\mathrm{K}_{\text {out }}$ values did not change with several sensitive analyses (data not shown).

The value of $I_{\max }$ decreased with repeated administration of NC-6004, such that the patients became increasingly tolerant with the $I_{\max }$ decreasing to half by day 25.4. The IOV was assumed in the PK/PD model and remained symmetrical over time (data not shown). The effect of hydration therapy was evaluated preliminarily and was shown to be associated with a $\triangle \mathrm{OFV}$ decrease by $2.556(P<0.110)$. The small number of patients (only five) precluded evaluation of its effect.

The hypothesis of $I_{\max }=1$ was evaluated preliminarily, and the $\triangle \mathrm{OVF}$ decreased by $1.77(P<0.183)$. However, the IOV for $I_{\max }$ was necessary and fixing $I_{\max }$ to 1 was thought not to be appropriate. The estimated $90 \%$ CI did not include one; however, it was decided that $\mathrm{I}_{\max }<1$ should remain in the model.

Figure 3 shows the correlation between the individual model prediction for eGFR and the observed eGFR. There was a good correlation, meaning that the model could sufficiently describe the decrease in eGFR after NC-6004 administration. There was no bias or symmetry over time in the weighted residuals (data not shown), so it was concluded that the model described the eGFR changes without bias for the whole period.

\section{Simulation of provability of acute kidney injury between NC-6004 and cisplatin}

The effect of cisplatin on acute kidney injury (AKI) was previously reported in patients with head and neck cancer [4]. All patients had received hydration in that study and the mean cisplatin dose was $99 \pm 9 \mathrm{mg} / \mathrm{m}^{2}$. According to the KDIGO definition of severity, of the 233 included patients, 158 (68\%) developed AKI; among these, 77 (49\%) developed stage 1 AKI, 55 (35\%) developed stage 2 AKI, and $26(16 \%)$ developed stage 3 AKI.

The simulation results for NC-6004 at a dose of $100 \mathrm{mg} / \mathrm{m}^{2}$ dose are compared with these results [4] in Fig. 4. Almost $70 \%$ of patients who received $100 \mathrm{mg} / \mathrm{m}^{2}$ of NC-6004 did not show AKI, and this ratio was double that shown with cisplatin. By comparison, the most common AKI grade was stage 1 with cisplatin treatment, with cisplatin causing a proportionally greater number of cases with AKI as the stage increased, when compared with NC-6004 (there were very few cases with stage 3 AKI). Although the percentage of patients with no AKI became less than $60 \%$ at an NC-6004 dose of $200 \mathrm{mg} / \mathrm{m}^{2}$, there were still few cases with stage 3 AKI and the overall safety profile did not change substantially from that for the $100 \mathrm{mg} / \mathrm{m}^{2}$ dose.
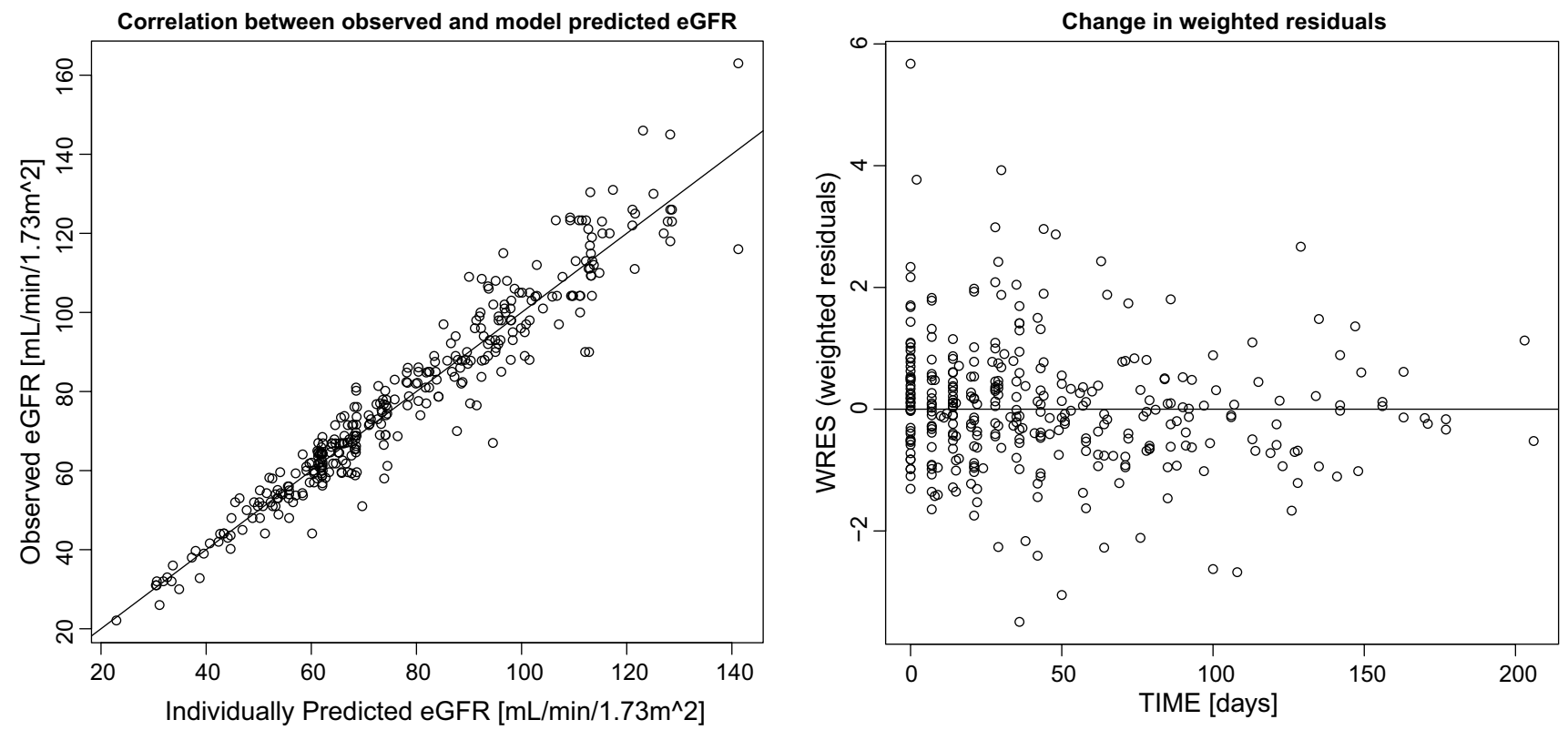

Fig. 3 Observed data vs. individual predicted data and weighted residuals vs. time for estimated glomerular filtration rate. $e G F R$ estimated glomerular filtration rate, IPRED individual predicted data, $D V$ observed data, WRES weighted residuals 


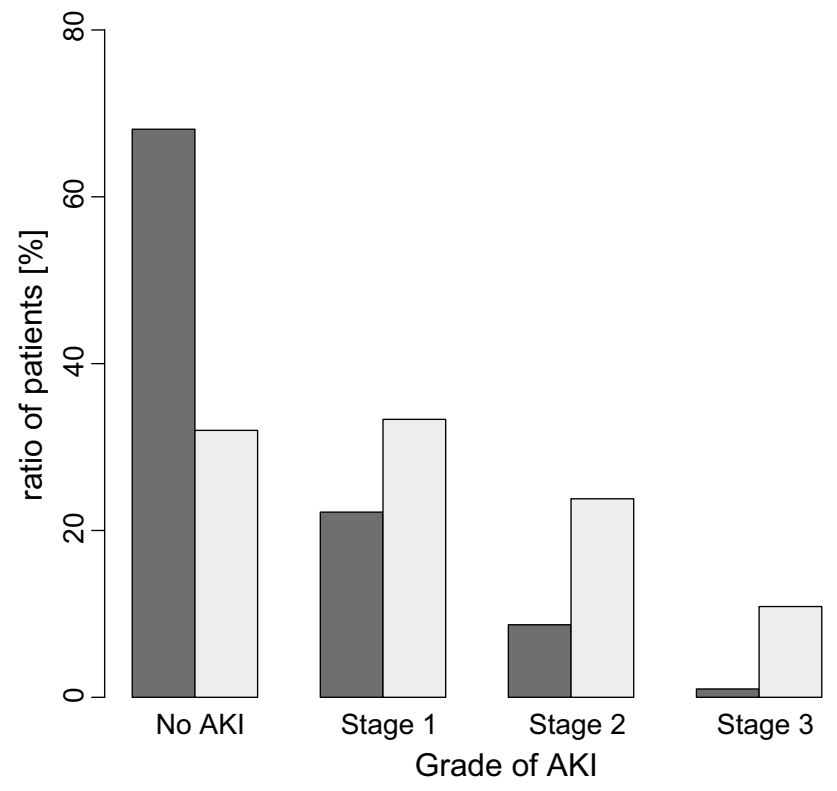

Fig. 4 Comparison of acute kidney injury between NC-6004 and cisplatin. Patients (\%) are shown by acute kidney injury (AKI) stage (black bar) for cisplatin $\left(99 \pm 9 \mathrm{mg} / \mathrm{m}^{2}\right)$ on days 3-4 [4] and (gray bar) as simulated for NC-6004 (100 mg) on day 6

\section{Discussion}

\section{Safety evaluation}

The safety profile of NC-6004 was expected to be similar to that of cisplatin. Therefore, neural, auditory, renal, and hematologic toxicity were considered potential AEs of NC-6004.

Concerning the occurrence of renal toxicity, $\mathrm{sCr}$ increased in two patients (16.7\%; one given $60 \mathrm{mg} / \mathrm{m}^{2}$ and one given $90 \mathrm{mg} / \mathrm{m}^{2}$ ). Renal disorder was also observed in two patients in the $90 \mathrm{mg} / \mathrm{m}^{2}$ cohort $(16.7 \%)$, with renal impairment occurring in one patient in the $90 \mathrm{mg} / \mathrm{m}^{2}$ cohort $(8.3 \%)$. These renal effects were all considered related to NC-6004. But after adding prophylactic hydration therapy before and after treatment with NC-6004, no further renal toxicity events occurred. The detail of the renal toxicity profile was revealed by population PK/PD analysis, which confirmed that the frequency and severity of AKI were much lower than those reported for cisplatin.

There was evidence of hematologic toxicity, with neutropenia being observed in 10 patients $(83.3 \%$; three given $60 \mathrm{mg} / \mathrm{m}^{2}$ and seven given $90 \mathrm{mg} / \mathrm{m}^{2}$ ), thrombocytopenia in nine patients $\left(75.0 \%\right.$; three given $60 \mathrm{mg} / \mathrm{m}^{2}$ and six given $90 \mathrm{mg} / \mathrm{m}^{2}$ ), and leukopenia in 10 patients $(83.3 \%$; three given $60 \mathrm{mg} / \mathrm{m}^{2}$ and seven given $90 \mathrm{mg} / \mathrm{m}^{2}$ ). Causality was attributed to NC-6004 for all of these events. However, no cases of neutropenia or thrombocytopenia were observed in the NC-6004 monotherapy phase, with both only occurring later the next day after starting gemcitabine. According to its package insert, myelosuppression is the most common AE with gemcitabine [6], and the package insert for cisplatin also states that myelosuppression may occur during treatment [7]. Hematologic toxicities that more than doubled in incidence between cycle one and cycle two and were experienced by at least two patients were anemia, neutropenia, thrombocytopenia, and leukopenia in the $60 \mathrm{mg} / \mathrm{m}^{2}$ cohort and hemoglobin decrease, neutropenia, thrombocytopenia, and leukopenia in the $90 \mathrm{mg} / \mathrm{m}^{2}$ cohort.

Although a grade 1 peripheral nerve disorder was observed in one patient $(8.3 \%)$, this neurotoxicity was not considered to be related to NC-6004. Hypersensitivity was also observed in one patient $(8.3 \%)$, but was judged to be an allergic reaction to the transfusion, and not to NC-6004. In addition, no auditory toxicity was observed. Adverse reactions showed the same pattern as AEs. Finally, six patients $(50.0 \%)$ from the safety population died within the 18-month follow-up period after the first dose. All of the deaths were considered to be caused by disease progression and were judged to be unrelated to NC-6004.

\section{Dosing: justification of the MTD and RD}

The MTD and RD of NC-6004 were determined to be 90 and $60 \mathrm{mg} / \mathrm{m}^{2}$, respectively, based on the protocol criteria. However, the RD was determined to be $90 \mathrm{mg} / \mathrm{m}^{2}$ for the following reasons after further discussion with the sponsor: (1) three of the four observed DLTs were considered typical, manageable hematologic toxicities of gemcitabine or cisplatin, and (2) the remaining DLT of cisplatin was shown to be controllable by prophylactic hydration.

\section{Population $P K$ and $P K / P D$ analyses}

The PK profile of NC-6004 was not influenced by ethnicity, i.e., difference between Japanese and Caucasians with population $\mathrm{PK}$ analysis.

The PK/PD model also explained the time course for eGFR after the first dose, as well as the significant decrease in amplification after repeated administrations. The eGFR decrease was thought to reach saturation at $100 \mathrm{mg} / \mathrm{m}^{2}$. Finally, although cisplatin had been reported to cause AKI in almost $70 \%$ of patients, the $\mathrm{PK} / \mathrm{PD}$ simulation indicated that NC-6004 caused AKI in only 30\% of patients. Most patients showed stage I AKI overall, but while there were high levels of stage II and III AKI with cisplatin, the distribution of AKI shifted in favor of no AKI or milder AKI with NC-6004 therapy.

\section{Compliance with ethical standards}

Funding This study was funded by NanoCarrier. 
Conflict of interest Mitsunori Harada, Kenichiro Naito, Naoto Hayashi, and Atsuhiro Masada are employees of NanoCarrier Co., Ltd. Toshihiko Doi received research funding from NanoCarrier Co., Ltd. The other authors have no conflicts of interest.

Ethical approval All procedures performed in studies involving human participants were in accordance with the ethical standards of the institutional and/or national research committee and with the 1964 Helsinki Declaration and its later amendments or comparable ethical standards.

Informed consent Informed consent was obtained from all individual participants included in the study.

Open Access This article is distributed under the terms of the Creative Commons Attribution 4.0 International License (http:// creativecommons.org/licenses/by/4.0/), which permits unrestricted use, distribution, and reproduction in any medium, provided you give appropriate credit to the original author(s) and the source, provide a link to the Creative Commons license, and indicate if changes were made.

\section{References}

1. Uchino H, Matsumura Y, Negishi T et al (2005) Cisplatin incorporating polymeric micelles (NC-6004) can reduce nephrotoxicity and neurotoxicity of cisplatin in rats. $\mathrm{Br} \mathrm{J}$ Cancer 93:678-687

2. Matsumura Y, Maeda H (1986) A new concept for macromolecular therapeutics in cancer chemotherapy: mechanism of tumoritropic accumulation of proteins and the antitumor agent SMANCS. Cancer Res 46:6387-6392

3. Sharma A, Jusko W (1998) Characteristics of indirect pharmacodynamic models and applications to clinical drug responses. Br J Clin Pharmacol 45:229-239

4. Bhat ZY, Cadnapaphornchai P, Ginsburg K, Sivagnanam M, Chopra S, Treadway CK (2015) Understanding the risk factors and long-term consequences of cisplatin-associated acute kidney injury: an observational cohort study. PLoS One 10:e0142225

5. Marcello T et al (2007) Short hydration regimen and nephrotoxicity of intermediate to high-dose cisplatin-based chemotherapy for outpatient treatment in lung cancer and mesothelioma. Tumori 93:138-144

6. Package insert of Gemsar [JAPAN], vol. 15, October 2013

7. Package insert of Randa [JAPAN], August 2014 